\title{
Polynomials counting nowhere-zero chains in graphs
}

\author{
Martin Kochol* \\ MU SAV \\ Bratislava, Slovakia \\ kochol@mat.savba.sk
}

Submitted: May 19, 2021; Accepted: Jan 17, 2022; Published: Jan 28, 2022

(C) The author. Released under the CC BY-ND license (International 4.0).

\begin{abstract}
We introduce polynomials counting nowhere-zero chains in graphs - nonhomogeneous analogues of nowhere-zero flows. For a graph $G$, an Abelian group $A$, and $b: V(G) \rightarrow A$, let $\alpha_{G, b}$ be a mapping from $\Lambda(G)$ (a family of vertex sets of connected subgraphs of $G$ satisfying an additional condition) to $\{0,1\}$ such that for each $X \in \Lambda(G), \alpha_{G, b}(X)=0$ if and only if $\sum_{v \in X} b(v)=0$. We prove that there exists a polynomial function $F(G, \alpha ; k)\left(\alpha=\alpha_{G, b}\right)$ of $k$ such that for any Abelian group $A^{\prime}$ of order $k$ and each $b^{\prime}: V(G) \rightarrow A^{\prime}$ satisfying $\alpha_{G, b^{\prime}}=\alpha, F(G, \alpha ; k)$ equals the number of nowhere-zero $A^{\prime}$-chains $\varphi$ in $G$ having boundaries equal to $b^{\prime}$. In particular $F(G, \alpha ; k)$ is the flow polynomial of $G$ if $\alpha(X)=0$ for each $X \in \Lambda(G)$. Finally we characterize $\alpha$ for which $F(G, \alpha ; k)$ is nonzero and show that in this case $F(G, \alpha ; k)$ has the same degree as the flow polynomial of $G$.
\end{abstract}

Mathematics Subject Classifications: 05C31, 05C21, 05C15

\section{Introduction}

The graphs considered in this paper are finite and unoriented. Multiple edges and loops are allowed. If $G$ is a graph, then $V(G)$ and $E(G)$ denote the sets of vertices and edges of $G$, respectively. With each edge of $G$ there are associated two distinct arcs (see [10]). Arcs on distinct edges are distinct. If an arc on an edge is denoted by $x$ the other is denoted by $x^{-1}$. If the ends of an edge $e$ of $G$ are vertices $u$ and $v$, one of the arcs on $e$ is said to be directed from $u$ to $v$ and the other one is directed from $v$ to $u$. Let $D(G)$ denote the set of arcs on $G$. If $v \in V(G)(X \subseteq V(G))$, then the set of arcs of $G$ directed out from $v$ (resp. $X)$ is denoted by $\omega_{G}(v)\left(\operatorname{resp} . \omega_{G}(X)\right)$.

\footnotetext{
*Partially supported by grant VEGA 2/0042/22.
} 
If $G$ is a graph and $A$ is an Abelian group, then a nowhere-zero $A$-chain in $G$ is a mapping $\varphi$ from $D(G)$ to $A \backslash\{0\}$ such that $\varphi\left(x^{-1}\right)=-\varphi(x)$ for every $x \in D(G)$. By the boundary of $\varphi$ we mean the mapping $\partial \varphi$ from $V(G)$ to $A$ such that $\partial \varphi(v)=\sum_{x \in \omega_{G}(v)} \varphi(x)$ for every $v \in V(G)$. By an easy computation (see [10, Proposition 2.1]) we can check that for any $X \subseteq V(G)$,

$$
\sum_{v \in X} \partial \varphi(v)=\sum_{x \in \omega_{G}(X)} \varphi(x)
$$

In particular, if $\left|\omega_{G}(X)\right|=0$ then $\sum_{v \in X} \partial \varphi(v)=0$ and if $\left|\omega_{G}(X)\right|=1$ then $\sum_{v \in X} \partial \varphi(v) \neq$ 0 . If $\partial \varphi(v)=0$ for every $v \in V(G)$, then we call $\varphi$ a nowhere-zero A-flow.

Nowhere-zero flows in graphs have been introduced by Tutte [15] as dual concept to graph coloring problems. It is well known that a planar graph is $k$-colorable if and only if its dual admits a nowhere-zero $A$-flow for any Abelian group of order $k$.

This paper is motivated by the facts that nowhere-zero flows are often studied in the framework of nowhere-zero chains (see cf. $[8,9,12,13]$ ) and that the number of nowherezero $A$-flows in a graph is evaluated by a polynomial function of $|A|$, the flow polynomial (see cf. $[1,2,3,4,7,14]$ ). Notice that flow polynomials are studied in framework of other combinatorial invariants, in particular the Tutte and characteristic polynomials - topics having applications not only in combinatorics, but also in coding theory, geometry, knot theory and physics (see $[6,5,16,17])$.

It is natural to ask whether there exist polynomial functions of $|A|$ that generalize flow polynomials and count nowhere-zero $A$-chains in graphs (but we are not aware whether this question appeared somewhere in the literature). If a graph consists from an isolated vertex $v$ and a loop, then the number of nowhere-zero $A$-chains $\varphi$ satisfying $\partial \varphi(v)=b_{v}$, $b_{v} \in A$, is either $|A|-1$ (if $b_{v}=0$ ), or 0 (if $b_{v} \neq 0$ ). With respect to similar arguments we need to consider so called " $\{0,1\}$-assignings" of certain sets of vertices and in the main result (Theorem 1) we show that the number of nowhere zero $A$-chains with boundaries corresponding to an assigning is evaluated by a polynomial function of $|A|$ that we call an assigning polynomial. As a consequence we can guarantee existence of group-valued chains if there exist chains with the same assigning and having values from a group of the same cardinality (Corollary 2). In the third section we decompose assigning polynomials and characterize assignings corresponding to nonzero polynomials. In the last section we present several examples and remarks about the topic.

\section{Assigning polynomials}

In this section we introduce the polynomial counting nowhere-zero chains in graphs.

For a graph $G$, let $c(G)$ denote the number of components of $G$ and $m(G)=|E(G)|-$ $|V(G)|+c(G)$. Denote by $\Lambda(G)$ the family of $X \subseteq V(G)$ such that $G[X]$ (the subgraph of $G$ induced by $X)$ is either a component of $G$ or $G[X]$ is connected and $c(G-X)=c(G)$. Notice that $\varnothing \in \Lambda(G)$. By an assigning of $G$ we mean any mapping $\alpha$ from $\Lambda(G)$ to $\{0,1\}$. We write $\alpha \equiv 0$ if $\alpha(X)=0$ for each $X \in \Lambda(G)$. 
Let $b: V(G) \rightarrow A$. By $\alpha_{G, b}$ we denote the assigning of $G$ such that for each $X \in \Lambda(G)$, $\alpha_{G, b}(X)=0$ if $\sum_{v \in X} b(v)=0$ and $\alpha_{G, b}(X)=1$ otherwise. We also say that $\alpha=\alpha_{G, b}$ is $G$ admissible. Let $\Phi_{G}(b)$ denote the set of nowhere-zero $A$-chains in $G$ satisfying $\partial \varphi(v)=b(v)$ for each $v \in V(G)$. If $\Phi_{G}(b) \neq \varnothing, b$ is called $G$-proper and $\alpha=\alpha_{G, b}$ is called a $G$-proper assigning of $G$. Clearly, each $G$-proper assigning is $G$-admissible, but not vice versa (more details are presented in the last section and Theorem 4).

For any edge $e$ of $G, G / e$ and $G-e$ denote the graph arising from $G$ after contracting and deleting $e$, respectively. Let $e \in E(G)$ have ends $v_{1}, v_{2}$. Denote by $v_{/ e}$ the vertex of $G / e$ arising from $v_{1}$ and $v_{2}$ after contracting $e$. For each $X \in \Lambda(G / e)$ denote by $X_{e}=\left(X \backslash\left\{v_{/ e}\right\}\right) \cup\left\{v_{1}, v_{2}\right\}$ if $v_{/ e} \in X$ and $X_{e}=X$ otherwise. Clearly, $X \in \Lambda(G / e)$ if and only if $X_{e} \in \Lambda(G)$. Thus for any assigning $\alpha$ of $G$ we can define $\alpha / e$ to be the assigning of $G / e$ such that $\alpha_{/ e}(X)=\alpha\left(X_{e}\right)$ for each $X \in \Lambda(G / e)$. Moreover $\Lambda(G-e) \subseteq \Lambda(G)$. Thus for any assigning $\alpha$ of $G$ we can define $\alpha_{-e}$ to be the assigning of $G-e$ such that $\alpha_{-e}(X)=\alpha(X)$ for each $X \in \Lambda(G-e)$. Clearly if $e$ is a loop (i.e, $v_{1}=v_{2}$ ), then $v_{/ e}=v_{1}=v_{2}, \Lambda(G)=\Lambda(G / e)=\Lambda(G-e)$, and $\alpha=\alpha_{/ e}=\alpha_{-e}$.

Theorem 1. Suppose that $\alpha$ is a $G$-admissible assigning of a graph $G$. Then there exists a polynomial function $F(G, \alpha ; k)$ of $k$ such that $F(G, \alpha ; k)=\left|\Phi_{G}(b)\right|$ for every Abelian group $A$ of order $k$ and every mapping $b: V(G) \rightarrow A$ satisfying $\alpha_{G, b}=\alpha . F(G, \alpha ; k)$ has degree $m(G)$ if $\alpha$ is $G$-proper and $F(G, \alpha ; k)=0$ otherwise. In the case $E(G)=\varnothing$ we have $F(G, \alpha ; k)=1$ if $\alpha$ is $G$-proper and $F(G, \alpha ; k)=0$ if $\alpha$ is not $G$-proper. Furthermore for any edge e of $G, \alpha_{/ e}$ and $\alpha_{-e}$ are $(G / e)$ - and $(G-e)$-admissible, respectively, and

$$
\begin{array}{llrl}
F(G, \alpha ; k) & =(k-1) F\left(G-e, \alpha_{-e} ; k\right), & & \text { if e is a loop, } \\
F(G, \alpha ; k) & =F\left(G / e, \alpha_{/ e} ; k\right)-F\left(G-e, \alpha_{-e} ; k\right), & & \text { otherwise. }
\end{array}
$$

Proof. We use induction by $|E(G)|$. If $|E(G)|=0$, then $m(G)=0, \Lambda(G)$ contains all subsets of $V(G)$ of cardinality at most 1 , and $\alpha$ is $G$-proper if and only if $\alpha \equiv 0$. Clearly, for any Abelian group $A$, there exist $(|A|-1)^{m(G)}=1$ nowhere-zero $A$-chains $\varphi$ in $G$. Thus $F(G, \alpha ; k)=(k-1)^{m(G)}=1$ if $\alpha \equiv 0$ and $F(G, \alpha ; k)=0$ otherwise, and the statement holds true in this case.

The induction step is trivial if $e$ is a loop of $G$ (as pointed out before, $\alpha=\alpha_{/ e}=\alpha_{-e}$ and $\Lambda(G)=\Lambda(G / e)=\Lambda(G-e)$ in this case).

Let $e$ be an edge of $G$ having different ends $v_{1}$ and $v_{2}$. Since $\alpha$ is $G$-admissible, there exist an Abelian group $A$ and $b: V(G) \rightarrow A$ such that $\alpha=\alpha_{G, b}$. Then $\alpha_{-e}=\alpha_{G-e, b}$ and $\alpha_{/ e}=\alpha_{G / e, \bar{b}}$ where $\bar{b}: V(G / e) \rightarrow A$ is defined so that $\bar{b}\left(v_{/ e}\right)=b\left(v_{1}\right)+b\left(v_{2}\right)$ and $\bar{b}(v)=b(v)$ if $v \neq v_{/ e}, v \in V(G / e)$. Thus $\alpha_{/ e}$ and $\alpha_{-e}$ are $(G / e)$ - and $(G-e)$-admissible, respectively.

Each $\varphi \in \Phi_{G / e}(\bar{b})$ can be considered as a nowhere-zero $A$-chain $\varphi^{\prime}$ in $G-e$ (considering $D(G / e)$ and $D(G-e)$ to be identical). Applying (1) for $\varphi$ and $\varphi^{\prime}$, we get that $\partial \varphi^{\prime}\left(v_{1}\right)=$ $b\left(v_{1}\right)+a$ and $\partial \varphi^{\prime}\left(v_{2}\right)=b\left(v_{2}\right)-a$ where $a \in A$. Then either $\varphi^{\prime} \in \Phi_{G-e}(b)$ (if $a=0$ ), or $\varphi^{\prime}$ can be extended into a unique nowhere-zero $A$-chain $\varphi^{\prime} \in \Phi_{G}(b)$ (if $a \neq 0$ ). Thus

$$
\left|\Phi_{G / e}(\bar{b})\right|=\left|\Phi_{G}(b)\right|+\left|\Phi_{G-e}(b)\right| .
$$


Let $\alpha$ be $G$-proper. Then by (3) also $\alpha / e$ is $(G / e)$-proper. By induction hypothesis and $(3),\left|\Phi_{G}\left(b^{\prime}\right)\right|=F\left(G / e, \alpha_{/ e} ; k\right)-F\left(G-e, \alpha_{-e} ; k\right)$ for any $b^{\prime}: V(G) \rightarrow A^{\prime},\left|A^{\prime}\right|=k$, satisfying $\alpha=\alpha_{G, b^{\prime}}$ (no matter whether $\alpha_{-e}$ is $(G-e)$-proper or not). Thus $\left|\Phi_{G}\left(b^{\prime}\right)\right|=F_{G, \alpha}(k)$ where $F_{G, \alpha}(k)$ equals $F\left(G / e, \alpha_{/ e} ; k\right)-F\left(G-e, \alpha_{-e} ; k\right)$. If $e$ is not a bridge in $G$, then $m(G)=m(G / e)=m(G-e)+1$ whence $F_{G, \alpha}(k)$ has degree $m(G)$. Let $e$ be a bridge in $G$. Then $m(G)=m(G / e)=m(G-e)$. Let $H$ be the component of $G$ containing $e$ and $H_{1}, H_{2}$ be the components of $H-e$. Using (1) and the fact that $\alpha$ is $G$-proper we have $\alpha(V(H))=0$ and $\alpha\left(V\left(H_{1}\right)\right)=\alpha\left(V\left(H_{2}\right)\right)=1$, whence by definition of $\alpha_{-e}$, $\alpha_{-e}\left(V\left(H_{1}\right)\right)=\alpha_{-e}\left(V\left(H_{2}\right)\right)=1$. Therefore by (1), $\alpha_{-e}$ is not $(G-e)$-proper and by the induction hypothesis, $F\left(G-e, \alpha_{-e} ; k\right)=0$. Thus $F_{G, \alpha}(k)=F\left(G / e, \alpha_{/ e} ; k\right)$ have degree $m(G)=m(G-e)$.

If $\alpha$ is not $G$-proper, then by induction hypothesis and (3), we have $F\left(G / e, \alpha_{/ e} ; k\right)=$ $F\left(G-e, \alpha_{-e} ; k\right)$, whence $F(G, \alpha ; k)=F\left(G / e, \alpha_{/ e} ; k\right)-F\left(G-e, \alpha_{-e} ; k\right)=0$.

$F(G, \alpha ; k)$ is called an $\alpha$-assigning polynomial of $G$.

Corollary 2. Suppose that $G$ is a graph, $A, A^{\prime}$ are Abelian groups of the same order and mappings $b: V(G) \rightarrow A, b^{\prime}: V(G) \rightarrow A^{\prime}$ satisfy $\alpha_{G, b}=\alpha_{G, b^{\prime}}$. Then $\left|\Phi_{G}(b)\right|=\left|\Phi_{G}\left(b^{\prime}\right)\right|$.

Proof. By Theorem 1, $\left|\Phi_{G}(b)\right|=F(G, \alpha ; k)=\left|\Phi_{G}\left(b^{\prime}\right)\right|$ where $\alpha=\alpha_{G, b}=\alpha_{G, b^{\prime}}$ and $k=|A|=\left|A^{\prime}\right|$.

Corollary 2 generalizes the well known property of nowhere-zero flows, namely that the number of nowhere-zero $A$-flows in a graph does not depend on the structure of $A$ but only on $|A|$.

The situation in Theorem 1 and Corollary 2 could be much simpler if we replace $\Lambda(G)$ by the powerset of $V(G)$. But in the last section we present an example of $b, b^{\prime}: V(G) \rightarrow A$ such that $\alpha_{G, b}$ equals $\alpha_{G, b^{\prime}}$ if these are considered as mappings form $\Lambda(G)$, but the equality does not hold if $\alpha_{G, b}$ and $\alpha_{G, b^{\prime}}$ are considered as mappings from $2^{V(G)}$. Thus we get $\left|\Phi_{G}(b)\right|=\left|\Phi_{G}\left(b^{\prime}\right)\right|$ by Corollary 2 , which is not possible if we consider $2^{V(G)}$ instead of $\Lambda(G)$. For this reason we need to consider $\Lambda(G)$ as small as possible, but, in the same time, $\Lambda(G)$ must be sufficiently rich so that we are able to prove Theorem 1 . Thus we choose a few technical definition of $\Lambda(G)$.

\section{Properties of assigning polynomials}

In this section we present a decomposition formula for assigning polynomials. Using the formula we characterize assignings corresponding to nonzero polynomials.

Suppose that $\alpha$ is a $G$-admissible assigning of a graph $G$. Then there exists an Abelian group $A$ and $b: V(G) \rightarrow A$ such that $\alpha_{G, b}=\alpha$. By a b-extension $\tilde{\alpha}_{G, b}$ (simply an extension $\tilde{\alpha}$ ) of $\alpha$ we mean a mapping from $2^{V(G)}$ to $\{0,1\}$ such that for each $X \subseteq V(G)$, $\tilde{\alpha}_{G, b}(X)=\tilde{\alpha}(X)=0$ if and only if $\sum_{v \in X} b(v)=0$. (In the following section we present an example when $\alpha_{G, b}=\alpha_{G, b^{\prime}}$ and $\tilde{\alpha}_{G, b} \neq \tilde{\alpha}_{G, b^{\prime}}$, i.e., $\alpha$ does not need to have a unique extension.) 
If $Y \subseteq E(G)$ and graph $G-Y$ has $c_{Y}$ components $G_{Y, 1}, \ldots, G_{Y, c_{Y}}$, then $\delta(Y, \tilde{\alpha})$ is defined so that

$$
\delta(Y, \tilde{\alpha})= \begin{cases}1 & \text { if } \tilde{\alpha}\left(G_{Y, 1}\right)=\cdots=\tilde{\alpha}\left(G_{Y, c_{Y}}\right)=0 \\ 0 & \text { otherwise }\end{cases}
$$

Let $<$ be a linear ordering of $E(G)$. We say that $Y \subseteq E(G)$ is <-compatible if for each circuit $C$ of $G, C \cap Y \neq\{\min (C)\}$ (considering circuits as edge sets of 2-regular connected subgraphs of $G)$. Denote by $\mathcal{E}(G,<)$ the set of all <-compatible subsets of $E(G)$. Clearly, no $<$-compatible set contains a loop (a circuit consisting of a single edge).

Theorem 3. Suppose that $\alpha$ is a $G$-admissible assigning of a graph $G, \tilde{\alpha}$ is an extension of $\alpha$, and let $<$ be a linear ordering of $E(G)$. Then

$$
F(G, \alpha ; k)=\sum_{Y \in \mathcal{E}(G,<)} \delta(Y, \tilde{\alpha})(-1)^{|Y|}(k-1)^{m(G-Y)} .
$$

Proof. We prove (5) applying induction on $\left|E(G) \backslash L_{G}\right|$ where $L_{G}$ denotes the set of loops of $G$. If $E(G)=L_{G}$, then $m(G)=|E(G)|$ and $\varnothing$ is the unique <-compatible subset of $E(G)$. Furthermore, $\delta(\varnothing, \tilde{\alpha})=1$ if $\alpha \equiv 0$ and $\delta(\varnothing, \tilde{\alpha})=0$ otherwise. Thus $F(G, \alpha ; k)=\delta(\varnothing, \tilde{\alpha})(k-1)^{m(G)}$ as claimed.

If $E(G) \neq L_{G}$, choose $e=\max \left(E(G) \backslash L_{G}\right)$ and define $\mathcal{E}^{+}=\{Y \in \mathcal{E}(G,<) ; e \notin Y\}$, $\mathcal{E}^{-}=\{Y \in \mathcal{E}(G,<) ; e \in Y\}$.

Let $Y \in \mathcal{E}(G / e,<)$ and $C$ be a circuit in $G$. If $e$ is a chord of $C$, then from $C$ arise two circuits $C_{1}^{\prime}, C_{2}^{\prime}$ in $G / e$ and $Y \cap C_{i}^{\prime} \neq\left\{\min \left(C_{i}^{\prime}\right)\right\}, i=1,2, \min (C) \in\left\{\min \left(C_{1}^{\prime}\right), \min \left(C_{2}^{\prime}\right)\right\}$, whence $Y \cap C \neq\{\min (C)\}$. If $e$ is not a chord of $C$, then from $C$ arise just one circuit $C^{\prime}$ in $G / e$ and $Y \cap C^{\prime} \neq\left\{\min \left(C^{\prime}\right)\right\}, \min (C)=\min \left(C^{\prime}\right)$, whence $Y \cap C \neq\{\min (C)\}$. Thus $Y \in \mathcal{E}(G,<)$ and $e \notin Y$, i.e., $\mathcal{E}(G / e,<) \subseteq \mathcal{E}^{+}$.

Let $Y \in \mathcal{E}^{+}$and $C$ be a circuit in $G / e$. Then $C$ indicates just one circuit $C_{e}$ in $G$ (where either $C=C_{e}$, or $C_{e}=C \cup\{e\}$ ). Since $Y \cap C_{e} \neq\left\{\min \left(C_{e}\right)\right\}$ and $e \notin Y$, we have $Y \cap C \neq\{\min (C)\}$. Thus $\mathcal{E}^{+} \subseteq \mathcal{E}(G / e,<)$ and $\mathcal{E}^{+}=\mathcal{E}(G / e,<)$.

If $Y \in \mathcal{E}(G-e,<)$, then for each circuit $C$ in $G-e, Y \cap C=(Y \cup\{e\}) \cap C \neq$ $\{\min (C)\}$. Furthermore, for each circuit $C^{\prime}$ in $G$ containing $e$ we have $e \in(Y \cup\{e\}) \cap C^{\prime}$, whence $(Y \cup\{e\}) \cap C^{\prime} \neq\left\{\min \left(C^{\prime}\right)\right\}$ (because by the choice of $e, e \neq \min \left(C^{\prime}\right)$ ). Thus $\mathcal{E}(G-e,<) \subseteq\left\{Y^{\prime} \backslash\{e\} ; Y^{\prime} \in \mathcal{E}^{-}\right\}$.

If $Y^{\prime} \in \mathcal{E}^{-}$, then for each circuit $C$ in $G-e,\left(Y^{\prime} \backslash\{e\}\right) \cap C=Y^{\prime} \cap C \neq\{\min (C)\}$, whence $\mathcal{E}(G-e,<)=\left\{Y^{\prime} \backslash\{e\} ; Y^{\prime} \in \mathcal{E}^{-}\right\}$.

Suppose that $\tilde{\alpha}_{/ e}$ and $\tilde{\alpha}_{-e}$ arise from $\tilde{\alpha}$ in a similar way as $\alpha_{/ e}$ and $\alpha_{-e}$ arise from $\alpha$, respectively. Clearly, $\tilde{\alpha}_{/ e}$ and $\tilde{\alpha}_{-e}$ are extensions of $\alpha_{/ e}$ and $\alpha_{-e}$, respectively (since $\tilde{\alpha}$ is an extension of $\alpha$ ).

It is an easy exercise to check that if $Y \in \mathcal{E}(G / e,<)=\mathcal{E}^{+}$, then $m((G / e)-Y)=$ $m(G-Y)$ (since $\left.e \notin L_{G}\right)$ and $\delta\left(Y, \tilde{\alpha}_{/ e}\right)=\delta(Y, \tilde{\alpha})$. Furthermore if $Y \in \mathcal{E}(G-e,<)=$ $\left\{Y^{\prime} \backslash\{e\} ; Y^{\prime} \in \mathcal{E}^{-}\right\}$, then $(G-e)-Y=G-(Y \cup\{e\})$ whence $\delta\left(Y, \tilde{\alpha}_{-e}\right)=\delta(Y \cup\{e\}, \tilde{\alpha})$. 
Thus by (2) and the induction hypothesis,

$$
\begin{gathered}
F(G, \alpha ; k)=F\left(G / e, \alpha_{/ e} ; k\right)-F\left(G-e, \alpha_{-e} ; k\right)= \\
\sum_{Y \in \mathcal{E}(G / e,<)} \delta\left(Y, \tilde{\alpha}_{/ e}\right)(-1)^{|Y|}(k-1)^{m((G / e)-Y)}- \\
\sum_{Y \in \mathcal{E}(G-e,<)} \delta\left(Y, \tilde{\alpha}_{-e}\right)(-1)^{|Y|}(k-1)^{m((G-e)-Y)}= \\
\sum_{Y \in \mathcal{E}^{+}} \delta(Y, \tilde{\alpha})(-1)^{|Y|}(k-1)^{m(G-Y)}- \\
\sum_{Y \in \mathcal{E}(G,<)} \delta\left(Y^{\prime}, \tilde{\alpha}\right)(-1)^{\left|Y^{\prime} \backslash\{e\}\right|}(k-1)^{m\left(G-Y^{\prime}\right)}= \\
\delta(Y, \tilde{\alpha})(-1)^{|Y|}(k-1)^{m(G-Y)},
\end{gathered}
$$

concluding the proof.

Notice that the summands from the right hand side of (5) can differ depending on the choice of $\tilde{\alpha}$ and $<$, but their sum is always $F(G, \alpha ; k)$.

Theorem 4. A G-admissible assigning $\alpha$ of a graph $G$ is $G$-proper if and only if

(a) for each component $H$ of $G, \alpha(V(H))=0$,

(b) for each bridge e, the components $H_{1}, H_{2}$ of $G-e$ containing the ends of e satisfy $\alpha\left(V\left(H_{1}\right)\right)=\alpha\left(V\left(H_{2}\right)\right)=1$.

Proof. Necessity follows directly from (1). To prove sufficiency, assume that (a) and (b) hold true. Let $Y \subseteq E(G)$ and $B_{G}$ denote the set of bridges of $G$. Then $m(G-Y)$ is smaller than $m(G)$ if $Y \backslash B_{G} \neq \varnothing$ and by (b), $\delta(Y, \tilde{\alpha})=0$ if $B_{G} \cap Y \neq \varnothing$. Thus for each linear ordering $<$ of $E(G)$ and each nonempty $Y \in \mathcal{E}(G,<)$, we have either $\delta(Y, \tilde{\alpha})=0$, or $m(G-Y)$ is smaller than $m(G)$. Clearly, $\varnothing \in \mathcal{E}(G,<)$ and by (a), $\delta_{\varnothing, \tilde{\alpha}}=1$. Thus the right hand side of $(5)$ is a sum of powers of $(k-1)$ such that exactly one of them (corresponding to $Y=\varnothing$ ) has the maximal possible degree $m(G)$. Hence $F(G, \alpha ; k) \neq 0$ and $\alpha$ is $G$-proper.

We show that if $\alpha \equiv 0$, then $F(G, \alpha ; k)$ is the flow polynomial of graphs (see cf. $[1,2,3,14])$.

Corollary 5. For any graph $G$, there exists a polynomial $F(G ; k)$ such that $F(G ; k)$ is equal to the number of nowhere-zero A-flows for any Abelian group $A$ of order $k$. Furthermore, $F(G ; k)$ has degree $m(G)$ if $G$ is bridgeless, $F(G ; k)=0$ if $G$ has a bridge, and for any edge e of $G$,

$$
\begin{array}{ll}
F(G ; k)=(k-1) F(G-e ; k), & \text { if e is a loop, } \\
F(G ; k)=F(G / e ; k)-F(G-e ; k), & \text { otherwise. }
\end{array}
$$


Proof. Clearly, $\alpha \equiv 0$ is $G$-admissible and by Theorem 4, it is $G$-proper if and only if $G$ is bridgeless. Thus the statement follows from Theorem 1 after setting $\alpha \equiv 0$.

If $\alpha \equiv 0$, then $\alpha=\alpha_{G, b}$ where $b(v)=0$ for each $v \in V(G)$, thus also $\tilde{\alpha} \equiv 0$ (i.e., $\tilde{\alpha}(X)=0$ for each $X \subseteq V(G))$. Then by $(4), \delta(Y, \tilde{\alpha})=1$ for each $Y \in \mathcal{E}(G,<)$, whence

$$
F(G ; k)=\sum_{Y \in \mathcal{E}(G,<)}(-1)^{|Y|}(k-1)^{m(G-Y)} .
$$

An alternative proof of this formula follows also from [11, Theorem 2].

Notice that formula (5) evaluates $F(G, \alpha ; k)$ as a sum of polynomials that form a subset of summands from the right hand side of (6) (that evaluates $F(G ; k)$ ). As pointed out in the proof of Theorem 4, for each $G$-proper assigning $\alpha$, the degree of $F(G, \alpha ; k)$ is determined by the summand $(k-1)^{m(G)}$ (corresponding to $\varnothing \in \mathcal{E}(G,<)$ ).

\section{Concluding remarks}

In this section we present several examples and remarks.

Example 1. Let $H=K_{2}, V(H)=\left\{v_{1}, v_{2}\right\}, E(H)=\{e\}$. Then $\Lambda(H)=2^{V(H)}$. Suppose that $\alpha$ is an assigning of $H$ such that $\alpha\left(\left\{v_{2}\right\}\right)=1$ and $\alpha(X)=0$ for all $X \in \Lambda(H)=2^{V(H)}$, $X \neq\left\{v_{2}\right\}$. It is easy to check that $\alpha$ is not $H$-admissible, $\alpha_{-e}$ is $H$-admissible but not $H$-proper, $\alpha_{/ e}$ is $H$-proper, $F\left(H-e, \alpha_{-e} ; k\right)=0$, and $F\left(H / e, \alpha_{/ e} ; k\right)=1$. If we set $F(H, \alpha ; k)=0$, then (2) is not valid any more. Thus it is necessary to formulate Theorem 1 for $G$-admissible assignings.

Example 2. Consider $b^{[i]}: V(H) \rightarrow \mathbb{Z}_{2}, i=1, \ldots, 3$, such that $b^{[1]}\left(v_{1}\right)=b^{[1]}\left(v_{2}\right)=1$, $b^{[2]}\left(v_{1}\right)=b^{[2]}\left(v_{2}\right)=0, b^{[3]}\left(v_{1}\right)=1, b^{[3]}\left(v_{2}\right)=0$. Thus $\alpha^{[i]}=\alpha_{H, b[i]}$ are $H$-admissible for $i=1, \ldots, 3$. By Theorem $4, \alpha^{[1]}$ and $\alpha_{/ e}^{[1]}$ are $H$ - and $(H / e)$-proper, respectively, $\alpha_{-e}^{[1]}$ is not $(H-e)$-proper, $F\left(H, \alpha^{[1]} ; k\right)=F\left(H / e, \alpha_{/ e}^{[1]} ; k\right)=1$, and $F\left(H-e, \alpha_{-e}^{[1]} ; k\right)=0$. On the other hand $\alpha^{[2]}$ is not $H$-proper, $\alpha_{/ e}^{[2]}$ and $\alpha_{-e}^{[2]}$ are $(H / e)$ - and $(H-e)$-proper, respectively, $F\left(H / e, \alpha_{/ e}^{[2]} ; k\right)=F\left(H-e, \alpha_{-e}^{[2]} ; k\right)=1$ and $F\left(H, \alpha^{[2]} ; k\right)=0$. Finally $\alpha^{[3]}, \alpha_{/ e}^{[3]}$, and $\alpha_{-e}^{[3]}$ are not $H-,(H / e)-$, and $(H-e)$-proper, respectively, and $F\left(H, \alpha^{[3]} ; k\right)=F\left(H / e, \alpha_{/ e}^{[3]} ; k\right)=$ $F\left(H-e, \alpha_{-e}^{[3]} ; k\right)=0$.

We need $\tilde{\alpha}$ only to define $\delta(Y, \tilde{\alpha})$. For this reason, it would suffice to define $\tilde{\alpha}$ as mappings from the family $\Lambda_{\mathrm{c}}(G)$ of vertex sets of connected subgraphs of $G$. Clearly, $\Lambda(G) \subseteq \Lambda_{\mathrm{c}}(G) \subseteq 2^{V(G)}$. Notice that Theorem 1 remains valid if we consider $\alpha$ as mappings from $\Lambda_{\mathrm{c}}(G)$ or $2^{V(G)}$, but then we get weaker versions of Theorem 1 .

Example 3. Let $G=K_{4,1}, u$ be the vertex of degree $4, u_{1}, \ldots, u_{4}$ be the vertices of degree 1 , and $b, b^{\prime}: V(G) \rightarrow \mathbb{Z}_{5}$ so that $b\left(u_{1}\right)=b\left(u_{2}\right)=1, b\left(u_{3}\right)=b\left(u_{4}\right)=4, b^{\prime}\left(u_{1}\right)=\cdots=$ $b^{\prime}\left(u_{3}\right)=1, b^{\prime}\left(u_{4}\right)=2$, and $b(u)=b^{\prime}(u)=0$. Then $\Lambda(G)$ consists of $\varnothing, V(G),\left\{u_{i}\right\}$, and $V(G) \backslash\left\{u_{i}\right\}(i=1, \ldots, 4)$, and $\alpha_{G, b}=\alpha_{G, b^{\prime}}$. Furthermore, $X=\left\{u_{1}, u_{4}, u\right\} \in \Lambda_{\mathrm{c}}(G) \backslash \Lambda(G)$ and $0=\sum_{v \in X} b(v) \neq \sum_{v \in X} b^{\prime}(v)$. Thus by Theorem 1 we have $\left|\Phi_{G}(b)\right|=\left|\Phi_{G}\left(b^{\prime}\right)\right|$, but we cannot apply this implication if we consider $\alpha$ as mappings from $\Lambda_{\mathrm{c}}(G)$ in Theorem 1 . 
Example 4. Let $C$ be a circuit of order 6 with consecutive vertices $v_{1}, \ldots, v_{6}$ and $b, b^{\prime}$ : $V(C) \rightarrow \mathbb{Z}_{5}$ so that $b\left(v_{1}\right)=\cdots=b\left(v_{3}\right)=1, b\left(v_{4}\right)=\cdots=b\left(v_{6}\right)=4, b^{\prime}\left(v_{2}\right)=2$, $b^{\prime}\left(v_{5}\right)=3$, and $b^{\prime}\left(v_{i}\right)=b\left(v_{i}\right)$ for $i \in\{1,3,4,6\}$. We can check that $\alpha_{C, b}=\alpha_{C, b^{\prime}}$, but $0=b\left(v_{1}\right)+b\left(v_{5}\right) \neq b^{\prime}\left(v_{1}\right)+b^{\prime}\left(v_{5}\right)$. Thus $\alpha_{C, b}=\alpha_{C, b^{\prime}}$ and $\tilde{\alpha}_{C, b} \neq \tilde{\alpha}_{C, b^{\prime}}$. By Theorem 1 we have $\left|\Phi_{G}(b)\right|=\left|\Phi_{G}\left(b^{\prime}\right)\right|$, but we cannot apply this implication if we consider $\alpha$ as mappings from $2^{V(G)}$ in Theorem 1. In fact we can check that for each $X \in \Lambda_{\mathrm{c}}(G), \sum_{v \in X} b(v)=0$ if and only if $\sum_{v \in X} b^{\prime}(v)=0$. Thus we get $\left|\Phi_{G}(b)\right|=\left|\Phi_{G}\left(b^{\prime}\right)\right|$ if we consider $\alpha$ as mappings from $\Lambda_{\mathrm{c}}(G)$ in Theorem 1.

In the following example we show that assumption $\alpha_{G, b}=\alpha_{G, b^{\prime}}$ cannot be deleted from Corollary 2. In fact we show that $F(G, \alpha ; k)$ can differ from $F\left(G, \alpha^{\prime} ; k\right)$ if $\alpha(X) \neq \alpha^{\prime}(X)$ for a unique $X \in \Lambda(G)$ (which, furthermore, has cardinality $>1$ ).

Example 5. Let $C$ be a circuit of order 6 with consecutive vertices $v_{1}, \ldots, v_{6}$ and $b, b^{\prime \prime}$ : $V(C) \rightarrow \mathbb{Z}_{5}$ such that $b\left(v_{1}\right)=\cdots=b\left(v_{3}\right)=1, b\left(v_{4}\right)=\cdots=b\left(v_{6}\right)=4, b^{\prime \prime}\left(v_{3}\right)=3$, $b^{\prime \prime}\left(v_{4}\right)=2$, and $b^{\prime \prime}\left(v_{i}\right)=b\left(v_{i}\right)$ for $i \in\{1,2,5,6\}$. Now $\alpha_{C, b} \neq \alpha_{C, b^{\prime \prime}}$ because $X=$ $\left\{v_{1}, v_{2}, v_{3}\right\} \in \Lambda(C)$ but $\sum_{i=1}^{3} b^{\prime \prime}\left(v_{i}\right)=0 \neq \sum_{i=1}^{3} b\left(v_{i}\right)$ (it is easy to check that $X$ and $V(C) \backslash X$ are the unique elements of $\Lambda(C)$ such that $\left.\alpha_{C, b}(X) \neq \alpha_{C, b^{\prime \prime}}(X)\right)$. Let $x_{i}$ denote the arc directed from $v_{i}$ to $v_{i+1}$ (considering the indices mod 6). Let $\varphi \in \Phi_{C}(b)$. If $\varphi\left(x_{1}\right)=$ 2 , then we can check that $\left(\varphi\left(x_{1}\right), \ldots, \varphi\left(x_{6}\right)\right)=(2,3,4,3,2,1)$ and $\varphi\left(x_{1}\right)$ cannot equal 3 (resp. 4, or 1 ) because then $\varphi\left(x_{3}\right)$ (resp. $\varphi\left(x_{2}\right)$, or $\varphi\left(x_{6}\right)$ ) equals 0 . Thus $\left|\Phi_{C}(b)\right|=1$. Similarly if $\varphi^{\prime \prime} \in \Phi_{C}\left(b^{\prime \prime}\right)$ and $\varphi^{\prime \prime}\left(x_{1}\right)=2\left(\operatorname{resp} . \varphi^{\prime \prime}\left(x_{1}\right)=3\right)$, then $\left(\varphi^{\prime \prime}\left(x_{1}\right), \ldots, \varphi^{\prime \prime}\left(x_{6}\right)\right)=$ $\left.(2,3,1,3,2,1)\left(\operatorname{resp} . \quad\left(\varphi^{\prime \prime}\left(x_{1}\right), \ldots, \varphi^{\prime \prime}\left(x_{6}\right)\right)=(3,4,2,4,3,2)\right)\right)$ and $\varphi^{\prime \prime}\left(x_{1}\right)$ cannot equal 4 (resp. 1), because then $\varphi^{\prime \prime}\left(x_{2}\right)$ (resp. $\varphi^{\prime \prime}\left(x_{6}\right)$ ) equals 0 . Hence $\left|\Phi_{C}\left(b^{\prime \prime}\right)\right|=2$, i.e., $\left|\Phi_{C}(b)\right| \neq$ $\left|\Phi_{C}\left(b^{\prime \prime}\right)\right|$. On the other hand considering $b^{\prime}$ as defined in Example 4, we have proved that $\alpha_{G, b}=\alpha_{G, b^{\prime}}$ and $\left|\Phi_{C}(b)\right|=\left|\Phi_{C}\left(b^{\prime}\right)\right|$.

By a leaf of a forest $T$ we mean any vertex of degree 1 or 0 . For each component $H$ of $F$, denote by $\ell(H)$ the set of leaves of $H$. By $\mathcal{L}(T)$ we mean the partition $\{\ell(H)\}$ of $\ell(T)$ where $H$ runs through the set of components of $T$. If $e$ is an edge of $H$, then $H-e$ consists of two subtrees $H_{1}, H_{2}$ of $H$, and denote by $\mathcal{L}(e)$ the partition $\left\{\ell\left(H_{1}\right) \cap \ell(H), \ell\left(H_{2}\right) \cap \ell(H)\right\}$ of $\ell(H)$ (notice that $\ell\left(H_{1}\right), \ell\left(H_{2}\right) \subseteq \ell(H)$ and $\mathcal{L}(e)=\left\{\ell\left(H_{1}\right), \ell\left(H_{2}\right)\right\}$ if $e$ is not incident with a vertex of degree 2$)$.

Assume that $G$ is a graph where $V(G)=\left\{v_{1}, \ldots, v_{n}\right\}$ has cardinality $n \geqslant 1$ and let $T$ be a forest having $n$ leaves $v_{1}^{\prime}, \ldots, v_{n}^{\prime}$. For $i=1, \ldots, n$, identify $v_{i}$ with $v_{i}^{\prime}$ into a new vertex $v_{i}^{\prime \prime}$. Denote the resulting graph by $G_{T}$. Let $A$ be an Abelian group. We say that a nowhere-zero $A$-chain $\varphi$ in $G$ is a nowhere-zero $(A, T)$-chain if $\sum_{v \in X} \partial \varphi(v)=0$ for each $X \in \mathcal{L}(T)$ and $\sum_{v \in Y} \partial \varphi(v) \neq 0$ for each edge $e$ of $T$ and $Y \in \mathcal{L}(e)$. It is easy to check that if $\varphi$ is a nowhere-zero $A$-flow in $G_{T}$ then the restriction $\psi$ of $\varphi$ into $D(G)$ is a nowhere-zero $(A, T)$-chain in $G$ and if $\psi$ is a nowhere-zero $(A, T)$-chain in $G$ then it can be extended into a unique nowhere-zero $A$-flow in $G_{T}$. Thus there is a one-to-one correspondence between the sets of nowhere-zero $A$-flows in $G_{T}$ and nowhere-zero $(A, T)$-chains in $G$. Therefore the flow polynomial $F\left(G_{T} ; k\right)$ of $G_{T}$ counts the number of nowhere-zero $(A, T)$-chains in $G$ for $k=|A|$.

For an Abelian group $A$ we say that $b: V(G) \rightarrow A$ is an $(A, T)$-mapping on $V(G)$ if $\sum_{v \in X} b(v)=0$ for each $X \in \mathcal{L}(T)$ and $\sum_{v \in Y} b(v) \neq 0$ for each edge $e$ of $T$ and $Y \in \mathcal{L}(e)$. 
Then $\varphi$ is a nowhere-zero $(A, T)$-chain in $G$ if and only if $\partial \varphi$ is an $(A, T)$-mapping on $V(G)$.

Consider $b, b^{\prime \prime}$ as defined in Example 5. Then $\left|\Phi_{C}(b)\right| \neq\left|\Phi_{C}\left(b^{\prime \prime}\right)\right|$. We can check that $b$ and $b^{\prime \prime}$ are $\left(\mathbb{Z}_{5}, T\right)$-mappings where $\mathcal{L}(T)=\left\{\left\{v_{1}^{\prime}, v_{6}^{\prime}\right\},\left\{v_{2}^{\prime}, v_{5}^{\prime}\right\},\left\{v_{3}^{\prime}, v_{4}^{\prime}\right\}\right\}$ ( $T$ consists of three copies of $\left.K_{2}\right)$. This example shows that the notion of $(A, T)$-mapping does not have an analogous application as presented in Corollary 2.

Suppose that $T$ is a forest such that exactly one component of $T$ is isomorphic either with $K_{2}$, or with $K_{1,3}$, and all other components are isolated vertices. It is easy to check that for each $(A, T)$-mapping $b$ on $V(G)$ we have $F\left(G, \alpha_{G, b} ; k\right)=F\left(G_{T} ; k\right)$. But in general we do not know whether for any assigning $\alpha$ of a graph $G$, the polynomial $F(G, \alpha ; k)$ can be evaluated by polynomials $F\left(G_{T} ; k\right)$ for some forests $T$.

\section{Acknowledgements}

The author thanks unknown referees for their comments.

\section{References}

[1] M. Beck, F. Breuer, L. Godkin, and J. L. Martin. Enumerating colorings, tensions and flows in cell complexes. J. Combin. Theory Ser. A, 122:82-106, 2014.

[2] M. Beck and T. Zaslavsky. Inside-out polytopes. Adv. Math., 205:134-162, 2006.

[3] F. Breuer and R. Sanyal. Ehrhart theory, modular flow reciprocity, and the Tutte polynomial. Math. Z., 270:1-18, 2012.

[4] B. Chen and R. P. Stanley. Orientations, lattice polytopes, and group arrangements II: modular and integral flow polynomials of graphs. Graphs Combin., 28:751-779, 2012.

[5] J.A. Ellis-Monaghan and I. Moffatt. The Tutte-Potts connection in the presence of an external magnetic field. Adv. in Appl. Math., 47:772-782, 2011.

[6] T. Brylawski and J. Oxley. The Tutte polynomial and its applications. In Matroid Applications (N. White, ed.), pages 123-225. Cambridge University Press, Cambridge, 1992.

[7] B. Jackson. Chromatic polynomials. In Topics in Chromatic Graph Theory (L. W. Beineke and R. J. Wilson, eds.), pages 56-72. Cambridge University Press, Cambridge, 2015.

[8] F. Jaeger, N. Linial, C. Payan, and M. Tarsi. Group connectivity of graphs - a nonhomogeneous analogue of nowhere-zero flow properties. J. Combin. Theory Ser. $B, 56: 165-182,1992$.

[9] T. Jensen and B. Toft. Unsolved graph colouring problems. In Topics in Chromatic Graph Theory (L. W. Beineke and R. J. Wilson, eds.), pages 327-357. Cambridge University Press, Cambridge, 2015. 
[10] M. Kochol. Superposition and constructions of graphs without nowhere-zero $k$-flows. European J. Combin., 23:281-306, 2002.

[11] M. Kochol. Interpretations of the Tutte and characteristic polynomials of matroids. J. Algebraic Combin., 53:1-9, 2021.

[12] H.-J. Lai, R. Luo, and C.-Q. Zhang. Integer flows and orientations. In Topics in Chromatic Graph Theory (L. W. Beineke and R. J. Wilson, eds.), pages 181-198. Cambridge University Press, Cambridge, 2015.

[13] L.M. Lovász, C. Thomassen, Y. Wu, and C.-Q. Zhang. Nowhere-zero 3-flows and modulo k-orientations. J. Combin. Theory Ser. B, 103:587-598, 2013.

[14] G. F. Royle. Recent results on chromatic and flow roots of graphs and matroids. In Surveys in Combinatorics 2009 (S. Huczynska, J. D. Mitchell, and C. M. RoneyDougal, eds.), pages 289-327. Cambridge University Press, Cambridge, 2009.

[15] W.T. Tutte. A contribution to the theory of chromatic polynomials. Canad. J. Math., 6:80-91, 1954.

[16] D.J.A. Welsh. Complexity: Knots, Colourings and Counting. Cambridge University Press, Cambridge, 1993.

[17] T. Zaslavsky. The Möbius function and the characteristic polynomial. In Combinatorial Geometries (N. White, ed.), pages 114-138. Cambridge University Press, Cambridge, 1987. 